\title{
Allier kastom et tabu au musée : gestion et exposition des objets du Vanuatu
}

Combine kastom and tabu at the museum: management and exhibition of objects from Vanuatu

\section{Marion Bertin}

\section{(2) OpenEdition}

Journals

Édition électronique

URL : http://journals.openedition.org/iss/1316

ISSN : 2306-4161

Éditeur

ICOM - International Council of Museums

Édition imprimée

Date de publication : 12 octobre 2019

Pagination : 41-55

ISBN : 978-92-9012-467-2

ISSN : 2309-1290

Référence électronique

Marion Bertin, «Allier kastom et tabu au musée : gestion et exposition des objets du Vanuatu », ICOFOM Study Series [En ligne], 47(1-2) | 2019, mis en ligne le 12 octobre 2019, consulté le 24 octobre 2019. URL : http://journals.openedition.org/iss/1316 


\section{Allier kastom et tabu au musée : gestion et exposition des objets du Vanuatu}

\section{Marion Bertin}

École du Louvre - Paris, France Université de La Rochelle - La Rochelle, France

ABSTRACT

Combine kastom and tabu at the museum: management and exhibition of objects from Vanuatu. Secret and sacred are extremely linked in Vanuatu. Some cultural rights precisely dictate and restrict how people can access to sacred objects and knowledges. These rules, as well as the traditions they are connected to, are also important for affirming identity inside this young state. This paper questions how to manage and exhibit those sacred objects in different settings, in the Vanuatu cultural center in Port-Vila (Vanuatu Kaljoral Senta), as well as in the case of temporary exhibitions organised by museums in Europe or in Pacific islands. Thus, we discuss the different significations of those objects and the possibility to create a cultural dialogue regarding their management. 
Keywords: Vanuatu, kastom, tabu, museum, cultural rights.

RÉsumé

Au Vanuatu, secret et sacré sont fortement liés. Des règlementations culturelles dictent avec précision les accès et restrictions de chacun concernant les objets et savoirs se rapportant au sacré. Ces codifications et les traditions qu'elles touchent ont une grande importance dans le cadre de l'affirmation identitaire de cette jeune république. Cet article aborde la gestion et l'exposition de tels objets dans différents contextes, au sein du centre culturel du Vanuatu à Port-Vila (Vanuatu Kaljoral Senta) d'une part et lors d'expositions temporaires organisées par des musées en Europe ou dans les îles du Pacifique d'autre part. Notre propos interroge ainsi les variations de sens et d'appréciation de ces objets sacrés et la possibilité d'un dialogue culturel autour de leur gestion.

Mots-clés : Vanuatu, kastom, tabu, musée, droits culturels.

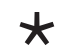

\section{Le Vanuatu : Big Men, tabu et kastom}

Archipel de quatre-vingt-trois îles, le Vanuatu est situé dans la partie sud de l'océan Pacifique, plus précisément en Mer de Corail. Après une concurrence entre la France et la Grande-Bretagne pour y établir leur souveraineté, il devient en 1906 un condominium franco-britannique portant le nom de « Nouvelles-Hébrides ». Malgré cette colonisation et la présence de missionnaires tant catholiques que protestants dans les îles, amenant de nombreuses conversions, les systèmes politiques et sociaux locaux, principalement fondés autour de sociétés de grades appelées « sociétés à Big Men » (Godelier, 2009), sont demeurés en place. Ces organisations occupent toujours une grande importance depuis l'accession à l'indépendance de la République du Vanuatu en 1980, qui marqua l'unification de ces diverses communautés jusqu'alors restées autonomes.

Ces sociétés hiérarchisées sont principalement masculines, pouvant être parfois féminines, mais procèdent d'une stricte division des genres. Elles donnent lieu à des passages de grades successifs, à la suite d'une première initiation, qui impliquent l'acquisition de capacités oratoires et le rassemblement de richesses, notamment des cochons à dents recourbées dont la croissance requiert une attention toute particulière. Le secret prédomine au sein de ces organisations où chaque nouveau grade permet à la personne concernée de s'approcher progressivement des ancêtres fondateurs de la lignée de la communauté. Ces ancêtres, ainsi que les esprits de la nature, sont au fondement de la perception 
et de la compréhension du sacré au Vanuatu, où réside donc un important lien avec le politique. Une stratification forte des savoirs intervient en parallèle et chaque passage de grade est synonyme de nouveaux apprentissages concernant la vie rituelle et politique, ainsi que les objets qui lui sont liés, qu'il en soit de leurs techniques de fabrication, de leurs usages ou de leur vision elle-même (Huffman, I996a). Cette organisation entraîne ainsi une codification forte des accès aux connaissances et aux productions matérielles ayant une dimension politique ou rituelle, qui sont soumises à des règles de tabu.

Le tabu codifie l'accés au sacré par le biais de prescriptions et de prohibitions. Il dicte ainsi les régles de conduite de chacun et permet de maintenir l'ordre cosmologique établi. (Bertin, 2018, p. 39).

Par ces indications, tout le monde ne sait pas tout ou bien sait différemment. Certains rituels demeurent largement secrets pour les personnes non initiées, qu'ils en soient des femmes, enfants ou toute autre personne exogène. Des réglementations culturelles strictes liées aux droits d'auteur, de propriété artistique et de disposition des objets fabriqués pour les rituels sont également attachées aux sociétés secrètes et plus largement aux productions du Vanuatu (Huffman, r996a).

Les sociétés actuelles du Vanuatu ne sauraient être comprises sans une seconde notion, apparue en lien avec les contestations à l'encontre du système colonial dans les années I970 : la kastom. Ce concept renvoie aux coutumes et traditions anciennes, mises en valeur et réaffirmées dans un contexte de revendications post-coloniales et la construction d'un État moderne unifié et indépendant (Geismar \& Tilley, 2003, p. I72). La kastom révèle donc une importance politique et suggère une approche dynamique et adaptative, plutôt qu'un ensemble de pratiques figées. Ainsi, elle est aujourd'hui largement alliée à la religion chrétienne (Huffman, 2013, p. 33). Elle englobe l'ensemble des sociétés et communautés de la jeune nation malgré leurs divergences et leurs diversités. Le respect des différents droits attachés à chaque groupe est cependant fondamental (Huffman, 2013). Les objets et productions matérielles ne sont pas au centre de la kastom, la perpétuation des savoirs et des techniques étant plus essentielle, mais ils tiennent une place qui ne doit pas être négligée. Ils occupent un rôle important dans la réappropriation des traditions anciennes, notamment grâce à ceux ayant été sauvegardés dans les musées européens.

$\mathrm{Si}$, dans un précédent article, nous avons questionné la gestion et l'exposition des objets plus largement océaniens dans des musées internationaux (Bertin, 20I8), nous nous interrogerons ici en particulier sur le cas des objets du Vanuatu. La persistance des traditions et l'importance de la kastom dans l'affirmation identitaire locale en font en effet un exemple intéressant à étudier précisément dans le cadre des relations entretenues entre musées et sacré. Si de nombreux 
objets issus des différentes îles du Vanuatu sont aujourd'hui conservés dans des institutions à travers le monde, l'existence d'un centre culturel à Port-Vila, le Vanuatu Kaljoral Senta (VKS) en bislama ${ }^{1}$, et son implication dans la sauvegarde des savoirs et des biens matériels, permettra de confronter différentes attitudes en regard des tabu.

Le Centre culturel du Vanuatu a ceci de particulier quill est conçu comme un lieu d'échange interactif entre les communautés des différentes îles et le musée comme une institution centrale. (Kasarhérou, Martin, Regenvanu \& Wilpert, 1996).

Il est « un espace communautaire, une maison publique de rencontres et un lieu pour conserver les objets adorés et commémorer l'histoire locale ${ }^{2}$ » (Alivizatou, 20I6, p. 9I). Grâce à un solide réseau d'interlocuteurs à travers l'archipel, le VKS est particulièrement impliqué dans la préservation, la protection et la diffusion de la kastom et porte une attention particulière à la gestion des savoirs et des objets tabu. Cette réflexion sur les modes de préservation et de présentation des objets du Vanuatu est à mettre en lien avec les débats et les projets de légalisation pour la protection des savoirs et des expressions traditionnels indigènes dans les îles du Pacifique, afin de limiter la dissémination des savoirs secrets (The Pacific Community, 1999), ainsi qu'une nouvelle conscience des musées quant à leur responsabilité en regard de ces protections (Brown, I998; Cummins, 2016).

Nous étudierons ainsi les modalités de gestion et d'exposition des objets tabu dans un premier temps au sein du VKS, sous la conduite des ni-Vanuatu euxmêmes, puis dans d'autres musées d'Europe et du Pacifique. Nous questionnerons finalement la possibilité d'un dialogue autour de cette notion et la manière de l'intégrer ou non dans l'espace muséal.

\section{Kastom et sacré au VKS}

Un musée est créé en 1956, au moment du cinquantième anniversaire du condominium, situé dans le centre-ville de Port-Vila, future capitale administrative du Vanuatu. Ses collections comportent des spécimens naturels et des objets collectés dans les îles de l'archipel, par l'Office de la recherche scientifique et technique outre-mer (ORSTOM) notamment. Dès ce moment, ses fonctions transcendent celles d'un musée, puisqu'y sont inclus un centre culturel, une

\footnotetext{
I. Une centaine de langues différentes sont parlées au Vanuatu. Le bislama est un pidgin dérivé de l'anglais qui permet une plus large compréhension entre les locuteurs. Il est une des trois langues officielles du Vanuatu, avec l'anglais et le français.

2. «A community space, a public meeting house and place for safekeeping cherished artefacts and commemorating local history» (traduction personnelle).
} 
bibliothèque nationale ainsi que des archives (Geismar \& Tilley, 2003, p. I72). Un conseil d'administration, dont les membres sont français, anglais et ni-vanuatu, est en charge de la gestion. Toutefois, peu de visiteurs ni-vanuatu fréquentent ce lieu (Bolton, 2003, p. 33-34). L'arrivée de l'ethnologue Kirk W. Huffman au poste de conservateur du musée en I979, en parallèle des recherches menées par des ethno-musicologues et des linguistes dans l'archipel, apporte quelques modifications avec une attention portée sur le patrimoine intangible toujours vivace et l'implication des populations ni-vanuatu elles-mêmes dans sa sauvegarde (Geismar \& Tilley, 2003, p. I72). Une importante équipe de bénévoles, les fieldworkers, est mise en place avec pour mission la collecte d'informations dans les diverses îles et leurs enregistrements sous forme audio et/ou visuelle (Sam, 1996). Ce programme a permis une prise de conscience des populations autour de leurs pratiques anciennes, au moment où émergeait le concept de kastom.

En novembre 1995, le VKS quitte le bâtiment qu'il occupait initialement pour une nouvelle construction située en face du Parlement de la République du Vanuatu et à proximité du Malvatumauri Nakamal, lieu de rassemblement coutumier du Conseil national des chefs, assemblée réunissant un ensemble de chefs élus par leurs homologues. À son inauguration, le bâtiment reçoit une double consécration, à la fois une bénédiction par l'Église et une cérémonie de plusieurs jours liée à la kastom (Huffman, 1996b), manifestant l'alliance de ces deux modes de pensée au Vanuatu. La présence d'objets sacrés originaires de l'ensemble des îles en fait un lieu tabu par excellence. Par cette nouvelle implantation et son aménagement répondant aux normes muséales mondiales, le VKS s'impose comme:

Un médiateur entre les conventions nationales de plus en plus génériques autour de la gestion et de linterprétation des objets et pléthore de divers intérêts locaux entourant les relations entre les personnes et les choses $^{1}$ (Geismar \& Tilley, 2003, p. 171).

\section{9}

Perdurant l'organisation en parapluie de ses origines, le VKS comprend un ensemble d'institutions nationales telles que le musée, la bibliothèque, les archives, la photothèque, tout en pilotant les nombreuses activités de collectes et de recherches dans les îles. Ces programmes, étendus à présent aux femmes ainsi qu'aux plus jeunes, occupent une place majeure dans les activités de l'institution. Ils sont essentiels pour la promotion et la diffusion de la kastom et de la culture du pays principalement à l'intérieur de l'archipel et pour permettre la postérité des informations (Sam, 1996). Un second centre culturel

\footnotetext{
I. «The Vanuatu Cultural Centre is a mediator between increasingly generic national conventions around the management and interpretation of objects, and a plethora of diverse local concerns around the relations between persons and things. » (traduction personnelle).
} 
a ainsi ouvert à Lakatoro, sur l'île de Malekula, en I99I, puis un troisième sur l'île de Tanna en 20ro. De plus, ces projets permettent véritablement de donner sens aux objets qui sont conservés et exposés par le VKS en les reliant directement aux pratiques sociales et rituelles des communautés et sociétés de grades. Cela permet en outre d'avoir une réelle connaissance des objets et des savoirs sensibles et de mieux en assurer la protection. Protection qui est donc multiple et nécessite leur préservation physique, le contrôle de leur accès, la diffusion restreinte des savoirs leur étant liés, afin de garantir en retour la protection des personnes. La distinction occidentale entre sujet et objet se révèle ici inopérante, les objets étant considérés comme vivants et capables d'incidences sociales.

Ainsi, les espaces d'exposition et de conservation du VKS s'organisent en tenant compte de ces modalités. Quatre espaces de réserves différents permettent d'ordonner les objets en fonction de leur sacralité et leur degré de tabu. L'accès de chacune de ces réserves est réglementé suivant le grade et l'initiation (Ralph Regenvanu, communication personnelle en date du 3 août 20I8). Les femmes ne peuvent s'en approcher, les objets les plus tabu étant souvent liés aux cérémonies masculines desquelles elles sont exclues. L'équipe du musée compte parmi ses membres des gardiens des savoirs et de la kastom qui veillent tout particulièrement sur ces objets et au respect des règles qui les entourent. La base de données, disponible uniquement en intranet depuis le VKS, répond également de ces exigences. Un «Database Committee » fut mis en place à partir de 2005 , réunissant des membres de chaque département du VKS et travaillant en étroites relations avec les intervenants bénévoles des communautés (Geismar \& Mohns, 20II, p. Si39). Différents niveaux d'accès, seize au total, ont ainsi pu être définis, selon le genre, la famille, le village et l'île d'origine des personnes amenées à consulter cette base de données (Geismar \& Mohns, 20II, p. SI49). Les objets les plus tabu ne peuvent être ni vus ni recherchés, car leur référencement empêche qu'ils soient trouvés lors d'une recherche. L'espace le plus restrictif de l'ensemble du VKS est celui réservé aux archives. Nommée « Tabu Room ", cette salle renferme les différents enregistrements, sonores ou visuels, les photographies et images liés aux rituels les plus secrets ayant fait l'objet de recherches par des fieldworkers ou confiés par les communautés elles-mêmes dans le but de protéger ces informations (Geismar \& Mohns, 20II, p. SI49-I50 ; Geismar \& Tilley, 2003, p. I79). Une copie est confiée aux personnes concernées par les pratiques référencées, qui en conservent la propriété intellectuelle et peuvent l'employer comme ressource culturelle (Geismar \& Mohns, 20II, p. SI39; Sam, I996, p. 298). Des objets peuvent également y être déposés par les communautés. Le VKS tient ainsi lieu de «banque de kastom ${ }^{\mathrm{I}}$ » (Geismar \& Mohns, 20II, p. SI49), dont il garantit la protection pour l'ensemble des îles de l'archipel. Les restrictions d'accès sont les mêmes pour tous les éléments déposés dans la Tabu Room, qui a pour rôle de préserver et de « contrôler les

I. « Bank of kastom » (traduction personnelle). 
effets des pouvoirs visuels de tels objets ${ }^{\mathrm{N}}{ }^{\prime}$ (Geismar \& Tilley, 2003, p. I79). Seuls les gardiens de la kastom peuvent ainsi avoir accès à cet espace (Geismar \& Tilley, 2003, p. 179).

D'après nos observations des vitrines présentées au musée national, les objets sont exposés dans la limite où ils ne sont pas trop sacrés ou tabu. Dans le cas d'objets étant tout de même liés à des rituels ou au sacré, les informations fournies par les cartels sont limitées. C'est le cas, entre autres, d'un rambaramp, mannequin funéraire réalisé à Malekula après la mort d'un grand homme et incorporant son crâne et ses os longs surmodelés dans une enveloppe de matériaux composites, ou encore de masques utilisés pour les cérémonies de passage de grade. La règle semble donc la suivante : soit on sait ce qu'est l'objet et donc les détails le concernant, soit on ne sait pas et le musée n'apportera guère plus d'informations.

Cela insinue que ce qui est, et ce qui peut être, exposé dans le hall d'exposition est fondamentalement structuré par des enjeux de droits et d'interdictions coutumiers². (Geismar \& Tilley, 2003, p. 175).

99

Une attention est également apportée à la stricte séparation des objets féminins et masculins, ce qui reflète la division des genres pratiquées au niveau rituel et politique. Ainsi, les productions textiles et vannières féminines sont mises à l'écart des vitrines liées aux cérémonies masculines (Geismar \& Tilley, 2003, p. 175). Les modalités d'exposition s'imposent comme le reflet de l'exigence des droits culturels traditionnels.

La césure entre hommes et femmes et le soin apporté au respect de la kastom et des tabu ne furent sans doute jamais plus marqués que lors de l'exposition The spirits of ancestors come back, ou Spirit Blong ol Bubu I Kam Bak en bislama, organisée en partenariat avec la Réunion des musées nationaux (Paris, France) ainsi que le Museum für Völkerkunde (Bâle, Suisse), et dont la première occurence eut lieu à Port-Vila du 28 juin au Io août 1996. Elle marquait le retour d'objets conservés dans des collections publiques européennes sur le sol de l'archipel. Le titre retenu pour l'étape de l'exposition au Vanuatu, imaginé par Ralph Regenvanu alors directeur du VKS (Christian Kaufmann, communication personnelle, en date du 25 novembre 20I8), traduit le rôle social des objets et leur considération par les populations du Vanuatu.

\footnotetext{
I. « Control the affective visual power of objects » (traduction personnelle).

2. "This intimates that what is, and can be, displayed in the exhibition hall is fundamentally structured by issues of customary rights and proscriptions. » (traduction personnelle).
} 
Il illustre le sens profond que ses divers maitres d'ouvre ont voulu lui donner en valorisant l'aspect affectif du retour momentané des objets sur leur lieu de création aprés de nombreuses années d'exil » (Coiffier, 1997, p. 513).

La sélection des objets amenés à voyager à Port-Vila fut opérée en grande partie par les fieldworkers, en tenant compte des objets que les habitants des îles voulaient voir. Certains de ces artefacts n'ont toutefois pu faire le déplacement, car empreints d'une trop grande puissance pour être demandés (Ralph Regenvanu, communication personnelle, en date du 3 août 20I8). Diverses cérémonies furent organisées, au moment du départ des objets vers le Pacifique, à leur arrivée à Port-Vila ou lors de l'inauguration de l'exposition (Huffman, I997; Vanuatu TV, 1996). Celles-ci, de la même manière que lors de l'ouverture du VKS, mêlaient kastom et christianisme (Huffman, 1997, p. 8-9). Plusieurs objets parmi les plus sacrés et les plus précieux pour les ni-Vanuatu avaient pu être transportés à Port-Vila, telles que des pierres à magie employées pour la fertilité et la pousse des dents de cochon. Elles font partie des objets les plus secrets du Vanuatu que les femmes et les non-initiés ne sont pas tenus de connaître ou d'apercevoir. Une «Tabu Haus » avait été aménagée dans un espace de la salle d'exposition du VKS, où seuls les hommes avaient autorisation de pénétrer (Huffman, 1997 , p. 5). La venue de ces objets n'était pas sans créer quelque crainte de la part des ni-Vanuatu les plus sensibles à leur puissance tandis que, à l'inverse, un débat avait été ouvert par les femmes remettant en cause la décision d'imposer cette restriction (Huffman, I997, p. II). Ce différend a permis d'envisager la création d'une «tabu room » réservée aux femmes pour d'autres expositions (Huffman, 1997, p. II). À notre connaissance, cette idée n’a jamais été concrétisée pour le moment. Le poids des tabu dans la vie publique n'est pas considéré d'un avis unanime. De plus, cette remise en question indique le privilège accordé aux savoirs et tapu masculins au sein de la kastom, bien qu'existent le Vanuatu National Council of Women, en plus d'un programme de recherche consacré aux femmes au VKS depuis I99I, autour du précepte "women have kastom too » (Bolton, 2003; Bolton, 2007).

\section{Appréhender le tabu hors du VKS}

L'exposition The spirits of ancestors come back, rebaptisée Vanuatu - Océanie. Arts des îles de cendre et de corail pour les étapes de Nouméa, Bâle et Paris ${ }^{1}$, a marqué un tournant dans l'appréhension et la gestion des objets du Vanuatu par les musées. Dans l'archipel, les communautés ont été particulièrement sensibles de

\footnotetext{
I. L'exposition a fait étape au musée territorial de Nouvelle-Calédonie à Nouméa du 3 septembre au 30 octobre 1996, puis au Museum für Völkerkunde de Bâle du I5 mars au Io août I997, avant de finir sa tournée au musée national des Arts d'Afrique et d'Océanie à Paris du zo septembre 1997 au 2 février 1998 .
} 
découvrir que des objets qui avaient disparu, car ils n'étaient plus localement fabriqués, avaient été conservés avec soin dans des musées internationaux (Huffman, I997, p. 2 ; Ralph Regenvanu, communication personnelle en date du 3 août 20r8). Les contacts et dialogues noués entre les institutions internationales et les communautés du Vanuatu sont à mettre plus largement en lien avec les efforts déployés autour de la prise en compte et la reconnaissance des prérogatives des communautés sources envers les objets qui leur sont attachés. La promulgation du Native American Graves Protection and Repatriation Act (NAGPRA) aux États-Unis en 1990 en est un exemple, promouvant le dialogue et obligeant les musées américains à retourner les biens sacrés et les restes humains aux communautés amérindiennes et hawaïennes. Les expositions menées autour de partenariats internationaux incluant les populations Māori, dès I984 (Mead, I984), ou les Kanaks en I990-I99I (De Jade et de nacre, 1990), ont également été déterminantes pour la conduite d'un projet conjoint avec le Vanuatu.

Dès le projet Vanuatu - Océanie, les liens avec les autorités coutumières du Vanuatu et avec les équipes du VKS ont eu une incidence sur la manière de concevoir l'exposition à l'international, tant dans la manière de présenter les objets que dans les savoirs transmis sur eux. Certains fieldworkers ni-vanuatu ont en effet questionné les organisateurs européens, parmi lesquels Joël Bonnemaison, Roger Boulay, Kirk Huffman et Christian Kaufmann, sur la gestion des objets hors du Vanuatu. Savoir si les femmes étaient autorisées à voir et à toucher les objets sacrés, ou encore si ces objets « étaient maintenus spirituellement heureux » (Huffman, I997, p. 2) ont fait partie des interrogations. Les pierres à magie, dont la dimension sacrée et secrète a déjà été soulignée, ont bénéficié d'une attention particulière pour leur installation au Museum für Völkerkunde (Christian Kaufmann, communication personnelle, en date du 20 décembre 20r8). Un espace clos leur fut consacré à Nouméa et à Bâle, avec la mention de leur sacralité, mais avec une plus grande flexibilité d'accès qu’à Port-Vila car les statuts des visiteurs étaient plus variés (Boulay \& Gonseth, I998, p. 48). L'écriture du catalogue s'est effectuée en considérant les droits et restrictions sur les savoirs traditionnels; les pierres à magie n'ont ainsi pas été commentées (Kaufmann, 1996, p.49). Les photographies de certains rituels n'ont par ailleurs pas été publiées. Ces précautions et la conscience des limites du travail d'ethnologue et de commissaire d'exposition sont assez récentes. Kirk Huffman note ainsi que la circulation secrète de l'ouvrage The Melanesians de Robert H. Codrington (I830-1922), qui comprend des descriptions très précises de cérémonies secrètes, dès sa publication en i89i dans les îles Banks et Torres, au nord de l'archipel du Vanuatu, aurait pu désintégrer les systèmes politiques et rituels locaux (Huffman, I996a, p. I90).

D'autres expositions temporaires consacrées au Vanuatu ont par la suite été à nouveau l'opportunité de nouer un dialogue avec les communautés afin de prendre connaissance et respecter leurs demandes. L'exposition Kastom. Art of Vanuatu présentée à la National Gallery of Australia (NGA) de Canberra de 
février à juin 2013 est un exemple intéressant à étudier. L'institution australienne a en effet mis en place depuis 2007 une politique concernant sa section «Arts du Pacifique » afin de valoriser les collaborations avec les îles voisines. Crispin Howarth, conservateur de cette collection et commissaire de ladite exposition Kastom, a ainsi fait appel à différentes sociétés du Vanuatu afin qu'elles puissent venir visiter l'exposition avant son ouverture. Cette consultation permettait de recueillir les avis de ces visiteurs sur les modalités de présentation ainsi que leurs propositions de modifications. Les commentaires ont principalement porté sur les restrictions dans l'accès aux différentes connaissances et principalement le clivage entre hommes et femmes (Howarth, 2016). Les discussions ont finalement conduit la NGA à mentionner plus explicitement sur les cartels et textes de salle la présence d'objets tabu, mais sans interdire ou limiter l'accès de certaines salles ou vitrines. Une cérémonie de levée des tabu a été organisée en amont du vernissage de l'exposition, permettant sa visite sans danger, à laquelle s'est adjointe une seconde cérémonie marquant la fin de cette manifestation et rétablissant le pouvoir des objets (Howarth, 20r6).

Ces différents dialogues ont ouvert la voie à des demandes de retour de certains objets. Les restes humains sont tout indiqués par de telles requêtes, tout particulièrement les rambaramp, effigies de grands hommes de l'île de Malekula. Marcellin Abong, directeur du VKS entre 2007 et 2014 venant lui-même de Malekula, avait ainsi profité du colloque organisé par le musée du quai Branly-Jacques Chirac en 2008 autour de la thématique des restes humains dans les musées pour formuler une demande de retour de ces mannequins funéraires.

Nous considérons toujours ces mannequins comme des personnes, ils ont toujours une vie, parce que nous croyons quil y a une vie aprés la mort, c'est pour cela que notre société n'a pas peur de la mort (Abong, 2008).

Le manque de respect dû aux tabu, ainsi que la nécessité d'honorer ces personnes par des funérailles, justifiaient cette demande, qui a pourtant été retirée quelques jours plus tard (Bécherel, 2008). Toutefois, des divergences d'opinion quant au retour des rambaramp existent, puisque d'autres personnes de Malekula ont refusé leur retour proposé par des musées australiens (Ralph Regenvanu, communication personnelle, en date du 3 août 20I8). C'est cependant un autre objet qui incarne certainement le plus les demandes de retour formulées par les populations ni-vanuatu : la pierre sacrée Lengnangulong, originaire du nord d'Ambrym. Acquise par l'ethnologue français Jean Guiart en 1949, elle est aujourd'hui exposée au Pavillon des Sessions du musée du Louvre et fait l'objet de demandes de retour au Vanuatu depuis les années i990, car les personnes l'ayant cédée n'en seraient pas les propriétaires coutumiers (DeBlock, 2017). 
Les gens d'Ambrym disent que la pierre Lengnangulong se promène maintenant la nuit dans les salles d'exposition du Louvre, en tentant de s'échapper et de rentrer chez elle au Vanuatu'

(DeBlock, 2017, p. 113).

99

Elle avait été présentée au VKS en 1996 où elle était visible au sein de la «Tabu Haus ", en tant qu'objet secret (Huffman, 1997, p. II). Une reproduction est toujours exposée au VKS (DeBlock, 20I7, p. II3). Cet exemple symbolise toute la complexité des liens qui unissent le sacré, les communautés du Vanuatu et les objets dont elles ont culturellement la possession et la responsabilité. Cependant, plutôt que les objets eux-mêmes, les ni-Vanuatu accordent souvent plus d'importance au retour d'images les représentant, essentielles pour la reproduction et la fabrication d'objets disparus et oubliés. La recréation de masques goulong de l'île de Malekula, dont les savoirs techniques avaient été perdus, grâce à l'apport de photographies prises par Marcellin Abong au musée national des Arts d'Afrique et d'Océanie à Paris (Alivizatou, 2016, p. 9I) est ici un cas exemplaire des apports d'une restitution visuelle pour les communautés d'origine des objets.

\section{Conclusion : secret et kastom au musée}

L'étude détaillée des objets du Vanuatu conservés dans des musées permet ainsi de mettre en lumière les rapports entretenus entre sacré et secret dans les communautés du Pacifique Sud et leurs conséquences pour la gestion et de l'exposition de telles productions matérielles. Cette analyse montre les limites du musée dans ses missions d'exposition et d'apports de connaissances (Derlon \& Jeudy-Ballini, 200I/2002). Elle questionne également la place accordée au dialogue avec les communautés et minorités culturelles et religieuses dans les pratiques des institutions muséales, ainsi que la reconnaissance des droits culturels. Dans les cas des communautés insulaires du Pacifique, les expositions temporaires, réunissant souvent de nombreuses institutions muséales en partenariat, sont particulièrement importantes. Enfin, cet examen interroge les différentes valeurs et significations accordées à un même objet selon son contexte d'exposition et de regard.

L'exposition The spirits of ancestors come back/Vanuatu - Océanie. Arts des îles de cendre et de corail cristallise l'ensemble de ces problématiques. Le sens de cette manifestation au Vanuatu, où elle symbolisait le retour d'objets sacrés sur leur terres d'origine, fut complètement différent en Nouvelle-Calédonie et en Europe, où l'accent était mis sur la présentation d'une culture peu connue des publics amenés à visiter l'exposition (Boulay \& Gonseth, I998). Les différences

\footnotetext{
I. « Ambrymese say the Lengnangulong stone now wanders around the night in the exhibition halls of the Louvre, trying to escape and come back home to Vanuatu» (traduction personnelle).
} 
de présentation s'en ressentent. La filiation entretenue entre les objets anciens et les productions actuelles, par le biais de la kastom, est déterminante. Roger Boulay notait ainsi dans le catalogue accompagnant l'exposition la frustration des ni-Vanuatu face à la présentation des objets en vitrine :

Voir les objets, et seulement les objets, dans notre exposition sera évidemment frustrant pour qui les a contemplés dans le contexte de leur présentation : je dis bien de présentation, de démonstration, seulement cela, sans aller décortiquer l'ensemble de la société. [...] Il faut admettre que la frustration est encore plus grande quand jessaie de décrire aux gens ici l'allure qu'aura chez nous l'exposition de leurs oeuvres et de celles de leur grands-péres - socles lisses, jolies lumieres, pas d'ombres naturelles, sons feutrés, crissements de pas sur les parquets cirés... (Boulay, 1996, p. 14).

9

C'est finalement toucher à l'essence même du sacré qui n'a d'existence que par la création d'une relation avec des personnes qui y croient. Certaines populations du Pacifique, tels que les communautés māori de Nouvelle-Zélande ou aborigènes d'Australie, ont réussi à imposer leurs voix à l'échelle internationale et jouissent à présent d'une autorité sur les objets attachés à leurs cultures conservés dans bon nombre de musées internationaux (Bertin, 20I8 ; McCarthy, 20II). Si des dialogues ont pu être noués, les voix des ni-Vanuatu sont cependant encore faibles pour que les mots de Kirk Huffman s'imposent au sein de la communauté muséale : «le monde extérieur n’a pas besoin de savoir, ou n'a pas le droit de savoir, et ces restrictions doivent être respectées ${ }^{\mathrm{I}}$ » (Huffman, I996b, p. 54).

\section{REMERCIEMENTS}

Cet article est amplement redevable à Roger Boulay, Kirk Huffman, Christian Kaufmann et Ralph Regenvanu qui ont accepté de partager leurs expériences et leurs souvenirs au Vanuatu Kaljoral Senta (VKS).

\section{Références}

Abong, M. (2008). Les restes humains ont-ils une place dans les musées aujourd'hui ?. Communication lors du colloque Des collections anatomique aux objets de culte : conservation et exposition des restes humains dans

I. « The outside world does not need to know, nor have the right to know, and these restrictions must be respected »(traduction personnelle). 
les musées, organisé par le musée du quai Branly, à Paris, France, les 22 et 23 février 2008.

Alivizatou, M. (2016). Intangible Heritage and the Museum: New Perspectives on Cultural Preservation. London, UK: Routledge.

Bertin, M. (2018). Tapu et musée : conserver et exposer des objets océaniens. Dans F. Mairesse (Éd.), Museology and the sacred (pp. 39-43). Paris, France : ICOFOM.

Brown, M. F. (I998). Can Culture be copyrighted ?. Current Anthropology, 39, 2, I93-222.

Bécherel, S. (2008, 28 février). Que faire des restes humains dans les musées?, France Inter, 28 février 2008. Page consultée le mercredi 20 février 20I9, au https://www.franceinter.fr/emissions/le-zoom-de-la-redaction/ le-zoom-de-la-redaction-28-fevrier-20o8.

Bolton, L. (2003). Unfolding the moon: enacting women's kastom in Vanuatu. Honolulu, USA: University of Hawai'i Press.

Bolton, L. (2007). Resourcing change: fieldwork, the Women's Cultural Project and the Vanuatu Cultural Centre. Dans Stanley, N. (Dir.). The Future of Indigenous Museums: Perspectives from the Southwest Pacific (pp. 23-37). London, UK: Berghan Books.

Boulay, R. (I996). Lettre d'Ambrym. Dans Musée territorial de Nouvelle-Calédonie, Museum für Völkerkunde, Musée national des arts d'Afrique et d'Océanie (Eds), Vanuatu - Océanie. Îles de cendre et de corail (pp.I3-I5). Paris, France : Réunion des musées nationaux \& ORSTOM.

Boulay, R., \& Gonseth, M.-O. (I998). L'expérience Vanuatu. Tsantsa, 3, 45-60.

Codrington, R. H. (I891). The Melanesians: Studies in their anthropology and folklore. Oxford, UK: The Clarendon Press.

Coiffier, C. (I997). Vanuatu, Océanie. Arts des îles de cendre et de corail, une exposition au musée national des arts d'Afrique et d'Océanie. Journal de la Société des Océanistes, I05, 213-2I5.

Cummins, A. (2016). Fragments for an epic memory, the politics (and geopolitics) of museums in the $2 \mathrm{I}^{\mathrm{st}}$ century. Communication lors $\mathrm{du} 4 \mathrm{O}^{\mathrm{e}}$ symposium d'ICOFOM, La Havane, Cuba, du 25 au 29 septembre 2016.

DeBlock, H. (2017). Property and ownership in Vanuatu: The Lengnangulong sacred stone from North Ambrym at the Pavillon des Sessions of the Musée du Louvre. Museum Anthropology, 4O, 2, III-I27.

De Jade et de nacre (I990). Paris, France : Réunion des musées nationaux.

Derlon, B., \& Jeudy-Ballini, M. (2001/2002). Le culte muséal de l'objet sacré. Gradhiva, 30/3I, 203-2II. 
Geismar, H., \& Mohns, W. (20II). Social relationships and digital relationships: rethinking the database at the Vanuatu Cultural Centre. Journal of the Royal Anthropological Institute, I7, I, SI33-SI55.

Geismar, H., \& Tilley, C. (2003). Negociating Materiality: international and local museums practices at the Vanuatu Cultural Centre and National Museum. Oceania, 73, I70-I88.

Godelier, M. (2009). La production des grands hommes : Pouvoir et domination masculine chez les Baruya de Nouvelle-Guinée. Paris, France : Flammarion.

Howarth, C. (2016). Indigenous protocoles in practice: experiences in the National Gallery of Australia. Communication présentée lors de la rencontre annuelle de la Pacific Arts Association - Europe, autour du thème Previous collections new obligations: the ongoing responsibilities of ethnographic museums, organisée au musée d'ethnographie de Genève, du 2 I au 23 novembre 2016.

Huffman, K. W. (I996a). Echanges, liens culturels et droits d'auteur : leur importance dans les arts du Vanuatu, dans Vanuatu - Océanie. Îles de cendre et de corail (pp. I90-203). Paris, France : Réunion des musées nationaux \& ORSTOM.

Huffman, K. W. (I996b). "Up and over": The opening of the Vanuatu Cultural Centre complex's new national museum building. Journal of the Pacific Arts Association, I3 \& I4, 47-56.

Huffman, K. W. (I997). Vanuatu Arts. Journal of the Pacific Arts Association, I5-I6, I-I4.

Huffman, K. W. (2013). 'Noho'n'dou yene nieve nungute I numuwo' hyene' - Respect is the foundation of life. Dans Howarth, C. (Ed.). Kastom. Art of Vanuatu (pp. 30-35). Coogee, Australia: New South Books.

Kasarhérou, E., Martin, J.-H., Regenvanu, R., \& Wilpert, C. (I996). Avant-propos. Dans Musée territorial de Nouvelle-Calédonie, Museum für Völkerkunde, Musée national des arts d'Afrique et d'Océanie (Eds), Vanuatu - Océanie. Îles de cendre et de corail (sans pagination). Paris, France : Réunion des musées nationaux \& ORSTOM.

Kaufmann, C. (1996). Arts de Vanuatu entre l'imagerie coutumière et forces d'expression. Dans Musée territorial de Nouvelle-Calédonie, Museum für Völkerkunde, Musée national des arts d'Afrique et d'Océanie (Eds), Vanuatu - Océanie. Îles de cendre et de corail (pp. I2-52). Paris, France : Réunion des musées nationaux \& ORSTOM.

McCarthy, C. (20II). Museums and Māori. Heritage professionals, indigenous collections, current practice. Wellington, Aotearoa New Zealand: Te Papa Press.

Mead, S. M. (I984). Te Maori: Maori Art from New Zealand Collections. New York, USA: Harry N. Abrams, Inc. 
Sam, J. (1996). Culture et audio-visuel : l'entreprise de documentation du VKS. Dans Musée territorial de Nouvelle-Calédonie, Museum für Völkerkunde, Musée national des arts d'Afrique et d'Océanie (Eds), Vanuatu - Océanie. Îles de cendre et de corail (pp. 298-299). Paris, France : Réunion des musées nationaux \& ORSTOM.

The Pacific Community (1999). Report of meeting: Symposium in the protection of traditional knowledge and expression of indigenous cultures in Pacifique islands. Nouméa, Nouvelle-Calédonie: SPC.

Vanuatu TV (1996). Yumi Takbaot Television Blong Vanuatu Vanuatu TV June 1996. "Spirit blong ol Bubu I kambak", YouTube. Page consultée le mercredi zo février 2019, au https://www.youtube.com/watch?time_ continue $=7 \& v=1$ DpYDgtQm_M. 